\title{
Matching 3D Shapes Using 2D Conformal Representations
}

\author{
Xianfeng $\mathrm{Gu}^{1}$ and Baba C. Vemuri ${ }^{2}$ \\ Computer and Information Science and Engineering, \\ Gainesville, FL 32611-6120, USA \\ $\{$ gu, vemuri\}@cise.ufl.edu
}

\begin{abstract}
Matching 3D shapes is a fundamental problem in Medical Imaging with many applications including, but not limited to, shape deformation analysis, tracking etc. Matching 3D shapes poses a computationally challenging task. The problem is especially hard when the transformation sought is diffeomorphic and non-rigid between the shapes being matched. In this paper, we propose a novel and computationally efficient matching technique which guarantees that the estimated nonrigid transformation between the two shapes being matched is a diffeomorphism.

Specifically, we propose to conformally map each of the two 3D shapes onto the canonical domain and then match these 2D representations over the class of diffeomorphisms. The representation consists of a two tuple $(\lambda, H)$, where, $\lambda$ is the conformal factor required to map the given $3 \mathrm{D}$ surface to the canonical domain (a sphere for genus zero surfaces) and $H$ is the mean curvature of the $3 \mathrm{D}$ surface. Given this two tuple, it is possible to uniquely determine the corresponding 3D surface. This representation is one of the most salient features of the work presented here. The second salient feature is the fact that $3 \mathrm{D}$ non-rigid registration is achieved by matching the aforementioned $2 \mathrm{D}$ representations.

We present convincing results on real data with synthesized deformations and real data with real deformations.
\end{abstract}

\section{Introduction}

Matching 3D shapes is a fundamental problem in computer vision with several applications in medical imaging, archeology, augmented reality and many others. Several 2D and 3D shape matching techniques have been developed in the past couple of decades in the field of computer vision and some have been successfully applied to medical imaging, shape retrieval etc. Since our focus in this paper is 3D shape matching, in the following, we will restrict our literature review to matching 3D shapes and refer the reader to some recent works [1]2,3] and the references therein for $2 \mathrm{D}$ shape matching.

3D shape matching has a rich history in the field of Computer Vision specifically in object recognition applications. A key ingredient in shape matching has been the choice of the shape representation scheme. One of the first 3D shape 
matching techniques involved the use of the curvature-based representations [4] 5], the modal based representations [6], harmonic images [7], spherical harmonic representation [8] and shape distributions [8], spline representations [9]. Very few of these representation along with the associated matching algorithms tackled non-rigid motions and when they did do so, the resulting motion was not a diffeomorphism. In some medical imaging applications, it is important to require that the estimated transformation be a diffeomorphism. Similar requirements are not uncommon in non-medical domains. More recently, there have been several techniques in literature 10 that yield a diffeomorphic mapping. These techniques however are relatively computationally expensive. In this paper, we propose a technique that is guaranteed to yield a diffeomorphic mapping in 3D and can be computed very efficiently via conformal matching of the corresponding $2 \mathrm{D}$ representation, described subsequently.

In [1], a technique for brain image warping was described and then applied to detect disease-specific patterns in [12. In [13], yet another diffeomorphic warping scheme developed by Christensen [14] was used to study hippocampal morphometry. In [15] Twining et al. developed a method to construct diffeomorphic representations of non-rigid registrations of medical images. Automatic neuroanatomical segmentation based on warping of 3D models is addressed in [16]. More recently, conformal brain mappings have been studied in [17] and [18]. While some of these techniques yield diffeomorphic warps, they are however computationally expensive. In this paper, we present a technique that yields diffeomorphic warps which are easy to compute and relatively inexpensive.

The rest of the paper is organized as follows: Section 2 contains some mathematical preliminaries. This is followed by the description of the conformal representation in section [3. Section 4 contains the mathematical formulation of the matching problem and the optimization method to find the best Möbius transformation. Experimental results on both synthetic and real data cases is presented in section 5 .

\section{Mathematical Framework for the Proposed Model}

We now present some definitions from differential geometry that are necessary to understand our shape representation.

Let $\phi: S_{1} \rightarrow S_{2}$ be a smooth map between two manifolds, define the local coordinates for $S_{1}$ and $S_{2}$ by $\left(x^{1}, x^{2}\right)$ and $\phi\left(x^{1}, x^{2}\right)=\left(\phi^{1}\left(x^{1}, x^{2}\right), \phi^{2}\left(x^{1}, x^{2}\right)\right)$. The first fundamental forms of $S_{1}$ and $S_{2}$ are: $d s_{1}^{2}=\sum_{i j} g_{i j} d x^{i} d x^{j}$ and $d s_{2}^{2}=$ $\sum_{i j} \tilde{g}_{i j} d x^{i} d x^{j}$. The pull back metric on $S_{1}$ induced by $\phi$ is then given by,

$$
\phi^{*} d s_{2}^{2}=\sum_{m n} \sum_{i j} \tilde{g}_{i j} \frac{\partial \phi^{i}}{\partial x_{m}} \frac{\partial \phi^{j}}{\partial x^{n}} d x^{m} d x^{n} .
$$

If there exists a positive function $\lambda\left(x^{1}, x^{2}\right)$, such that $d s_{1}^{2}=\lambda\left(x^{1}, x^{2}\right) \phi^{*} d s_{2}^{2}$, then we say $\phi$ is a conformal map between $S_{1}$ and $S_{2}$. Especially, if the map from $S_{1}$ to the local coordinate plane $\left(x_{1}, x_{2}\right)$ is conformal, we say that $\left(x_{1}, x_{2}\right)$ are the 
conformal coordinates of $S_{1}$. A 2-manifold $S$ is a Riemann surface, if there exists an atlas, such that, (1) each local chart forms a conformal coordinate system on $S$ and we treat each coordinate chart as an open set of the complex plane C. (2) The transition functions between charts are treated as complex functions, all of them being holomorphic. By the Riemann uniformization Theorem, all surfaces can be conformally embedded in a sphere, a plane or a hyperbolic space, wherein all the embeddings form special groups. The conformal parametrization continuously depends on the Riemannian metric tensor on the surface.

Surfaces can be represented as functions defined on their conformal coordinate systems. Thus, by using a conformal representation, the surface matching problems in $3 \mathrm{D}$ can be converted to matching of equivalent representations in 2D.

Suppose $S_{1}$ and $S_{2}$ are two surfaces we want to match and their conformal coordinate domains of $S_{1}$ and $S_{2}$ are $D_{1}$ and $D_{2}$ respectively. Let the conformal mapping from $S_{1}$ to $D_{1}$ be $\pi_{1}$, the one from $S_{2}$ to $D_{2}$ be $\pi_{2}$. Instead of finding the mapping $\phi$ from $S_{1}$ to $S_{2}$ directly, we want to find a map $\tilde{\phi}: D_{1} \rightarrow D_{2}$, such that the diagram 2 is commutable i.e., $\pi_{2}^{-1} \circ \tilde{\phi} \circ \pi_{1}=\phi$.

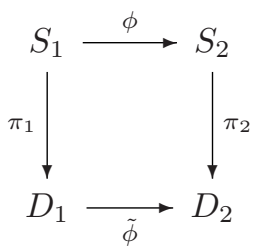

Thus, finding a diffeomorphic $\phi$ between $S_{1}$ and $S_{2}$ can be achieved by finding a diffeomorphism $\tilde{\phi}$ from $D_{1}$ to $D_{2}$ and then using the commutative diagram.

\section{The 2D Conformal Representation for Surfaces in 3D and Its Properties}

In this section we will introduce the methods to represent surfaces using their conformal coordinates. This representation preserves all the geometric information of the surface and maps all surfaces to the canonical $2 \mathrm{D}$ domains. If two surfaces are close to each other under the Housdorff metric, the $L^{2}$ norm between their conformal representations are also close. Conversely, if two surfaces have similar conformal representations, then they also have similar shapes in $R^{3}$. The key advantage of our conformal representation is that it is complete in the sense that it allows us to reconstruct the original surface fully from the representation. Thus, there is no loss of information in this representation unlike most others e.g., shape distributions [8] and others.

Suppose surface $S$ is mapped conformally to a canonical domain, such as the sphere. We can stereographically project the sphere to the complex plane, then use these conformal coordinates $(u, v)$ to parameterize $S$. Then, We can compute the following two maps directly from the position vector $S(u, v)$. First, the conformal factor map or stretch map is the conformal factor function $\lambda(u, v)$ 
defined on $(u, v)$, and conceptually represents the scaling factor at each point. Secondly, the mean curvature function.

$$
\begin{aligned}
\frac{\partial S}{\partial u} \times \frac{\partial S}{\partial v} & =\lambda(u, v) n(u, v) \\
\Delta S(u, v) & =H(u, v) n(u, v)
\end{aligned}
$$

where $\Delta$ is the Laplace-Beltrami operator defined on $S, n(u, v)$ is the normal function, $H(u, v)$ is the mean curvature, $\lambda(u, v)$ conformal factor function. The tuple $(\lambda, H)$ is the conformal representation of $S(u, v)$.

Theorem 1 Conformal Representation: If a surface $S(u, v)$ is parameterized by some conformal parameter $(u, v)$ on a domain $D$, then the conformal factor function $\lambda(u, v)$ and mean curvature function $H(u, v)$ defined on $D$ satisfy the Gauss and Codazzi equation. If $\lambda(u, v)$ and $H(u, v)$ are given, along with the boundary condition $\left.S(u, v)\right|_{\partial D}$, then $S(u, v)$ can be uniquely reconstructed.

The conformal maps between two genus zero closed surfaces form the so called Möbius transformation group. If we map the sphere to the complex plane using stereo-graphic projection, then all Möbius transformations $\mu: S^{2} \rightarrow S^{2}$ have the form

$$
\mu(z)=\frac{a z+b}{c z+d}, a d-b c=1, a, b, c, d \in C .
$$

Another important property of our representation is that it is very stable. If we slightly perturb a shape $S$, then its conformal representation $\lambda(u, v)$ will be perturbed only slightly. Hence, conformal representation is continuous and stable.

In summary, the conformal representation is intrinsic, continuous and stable, preserving all geometric information. It allows us to convert the problem of matching surfaces in $3 \mathrm{D}$ to the problem of matching the corresponding canonical $2 \mathrm{D}$ conformal representations.

\section{3D Shape Matching Formulation}

In this section, we present the mathematical formulation of the matching problem. Suppose we have two genus zero surfaces $S_{1}$ and $S_{2}$ embedded in $R^{3}$. Our goal is to find a diffeomorphism $\phi: S_{1} \rightarrow S_{2}$, such that $\phi$ minimizes the following functional,

$$
E(\phi)=\int_{D_{1}}\left\|S_{1}(u, v)-S_{2}(\phi(u, v))\right\|^{2} d u d v .
$$

If we want to find a conformal map $\phi$ between $S_{1}$ and $S_{2}$, we can restrict $\tilde{\phi}$ to be a Möbius transformation when $D_{1}$ and $D_{2}$ are spheres.

The position map/representation $S(u, v)$ is variant under rigid motion, and the conformal factor $\lambda(u, v)$ and mean curvature $H(u, v)$ are invariant under rigid 
motion. Therefore, it is most efficient to use $\lambda(u, v)$ and $H(u, v)$ for matching surfaces. The matching energy can therefore be defined as,

$E(\tilde{\phi})=\int_{D_{1}}\left\|\lambda_{1}(u, v)-\lambda_{2}(\tilde{\phi}(u, v))\right\|^{2} d u d v+\int_{D_{1}}\left\|H_{1}(u, v)-H_{2}(\tilde{\phi}(u, v))\right\|^{2} d u d v$.

This energy is minimized numerically to obtain the optimal $\tilde{\phi}$ and then the corresponding $\phi$ is obtained from the commutative diagram shown earlier. There are many numerical optimization algorithms in literature that can be used to minimize the energy defined above for diffeomorphic matching of functions defined on the plane e.g., the gradient descent method with adaptive step size, the Gauss-Newton method or the quasi-Newton method. In this paper, we use the quasi-Newton method to compute the optimal diffeomorphism $\tilde{\phi}$.

During the matching, the boundary condition and geometric constraints should be considered. A general diffeomorphism may not guarantee that corresponding points between two instances of the same shape will map to each other however it is a necessary condition. Thus, in order to make sure that this constraint is satisfied, we may choose certain landmark points on $S_{1}$ which we want to map to predefined points on $S_{2}$. This leads to a constrained diffeomorphic matching.

For genus zero closed surface, in order to find a Möbius transformation, three landmarks are enough. Suppose three landmarks are given as $\left\{z_{0}, z_{1}, z_{2}\right\}$, corresponding to $\left\{\tilde{z}_{0}, \tilde{z}_{1}, \tilde{z}_{2}\right\}$, then a Möbius transformation $\tilde{\phi}$ which maps all the landmarks can be represented in a closed form, we use $z_{i j}$ to denote $z_{j}-z_{i}$ , and $\tilde{z}_{i j}$ for $\tilde{z}_{j}-\tilde{z}_{i}$

$$
\tilde{\phi}(z)=\frac{\tilde{z}_{1}\left(z-z_{0}\right) z_{12} \tilde{z}_{02}-\left(z-z_{1}\right) z_{02} \tilde{z}_{12} \tilde{z}_{0}}{\left(z-z_{0}\right) z_{12} \tilde{z}_{02}-\left(z-z_{1}\right) z_{02} \tilde{z}_{12}}
$$

We match the landmarks first, then use that $\tilde{\phi}$ as the initial Möbius transformation, and optimize it to find the minimal matching energy.

\section{Experimental Results}

In order to test the performance of the matching algorithm, several experiments are carried out and described in this section. We use surfaces extracted from medical images for our testing. Their topologies are verified to be of genus zero. The shapes are represented by triangulated meshes in 3D which are subdivided using standard sub-division schemes in Computer Graphics to yield a smooth representation of the surfaces. These surfaces are conformally mapped to the canonical domain namely, the sphere, using the method introduced in 18] and these canonical mappings then form the input to our matching algorithm. We tested our algorithm with both synthetically generated deformations as well as real deformations. In the synthetic deformation cases, we applied known nonrigid deformations to a source shape to generate the target shape. The estimated deformation is then compared with the known ground truth deformation for 
several similarly generated data sets. The average and standard deviation of the error in estimated deformation was then computed over the entire source-target pairs of data. In the examples presented below, this error is quite small indicating the accuracy of our technique presented here.

\subsection{Synthetic Deformations Applied to Real Surfaces}

The deformation coefficients of $\phi$ are generated randomly using a Gaussian distribution. We used 20 sample deformations from the Guassian distribution of deformations to test our algorithm. As evident from the table 1, the mean and variance of the difference between $\phi$ and the reconstructed deformation $\hat{\phi}$ are very small indicating the accuracy of our algorithm.

In the next experiment, we present a cortical surface extracted from a human brain MRI image and represented using a triangular mesh as shown in 10 This cortical surface is then conformally mapped to a sphere as shown in (b) and (d). The conformal factor $\lambda$ is color-encoded as depicted in (a) and (b), the mean curvature map is also color-encoded as shown in (c) and (d). The lowest values of $\lambda$ and $H$ are represented by the blue color and the highest by the red color. Values in between span the color spectrum.

We now define a non-rigid motion denoted by $\phi: R^{3} \rightarrow R^{3}$ using affine maps and then apply it to the real anatomical surface extracted from the brain MRI scan. $\phi=\left(\phi^{1}, \phi^{2}, \phi^{3}\right)$ is represented as $\phi^{k}=\sum_{i} l_{i}^{k} x^{i}+t^{k}, i, j, k=1,2,3$. All coefficients of $\phi$ are randomly drawn from a Gaussian distribution.

The anatomical surface $S$ is deformed by a randomly generated $\phi$, then we match $S$ with $\phi(S)$ using the conformal representation. Using the algorithm described in the last section, we estimate $\phi$ and denoted it by $\hat{\phi}$. We computed the estimation error by comparing the original coefficients of $\phi$ and the estimated coefficients of $\hat{\phi}$. The results are depicted in the table 1 As evident, the estimates are very accurate. Figure 2 depicts one matching example. The deformation $\phi$ in 2 is

$$
\phi(x, y, z)=\left(\begin{array}{ccc}
5.0 & 0.10 & 0.2 \\
0.4 & 5.10 & 0.2 \\
0.4 & 0.6 & 4.9
\end{array}\right)\left(\begin{array}{l}
x \\
y \\
z
\end{array}\right)+\left(\begin{array}{c}
5.0 \\
10.0 \\
15.0
\end{array}\right)
$$

Table 1. Mean and variance of the computed deformations between the source and target shapes.

\begin{tabular}{|c|l|l|l|l||l|l|l|l||}
\hline \# of Tests & $\mu_{l_{i}^{k}}$ & $\sigma_{l_{i}^{k}}$ & $\mu_{t^{k}}$ & $\sigma_{t^{k}}$ & $\mu_{\hat{l}_{i}^{k}-l_{i}^{k}}$ & $\sigma_{\hat{l}_{i}^{k}-l_{i}^{k}}$ & $\mu_{\hat{t}^{k}-t^{k}}$ & $\sigma_{\hat{t}_{i}-t_{i}}$ \\
\hline 20 & 0.3 & 0.47 & 33.33 & 47.14 & $4.02 \mathrm{e}-4$ & $0.538 \mathrm{e}-4$ & $7.27 \mathrm{e}-4$ & $4.69 \mathrm{e}-4$ \\
\hline 20 & 1.9 & 2.21 & 10.00 & 4.08 & $2.7333 \mathrm{e}-2$ & $1.0607 \mathrm{e}-2$ & $3.2645 \mathrm{e}-2$ & $1.6135 \mathrm{e}-2$ \\
\hline 20 & 3.9 & 4.36 & 20.00 & 8.16 & $1.55339 \mathrm{e}-1$ & $9.3372 \mathrm{e}-2$ & $1.09864 \mathrm{e}-1$ & $7.1456 \mathrm{e}-2$ \\
\hline 20 & 1.8 & 2.21 & 10.00 & 4.08 & $2.7333 \mathrm{e}-2$ & $1.0607 \mathrm{e}-2$ & $3.2645 \mathrm{e}-2$ & $1.6135 \mathrm{e}-2$ \\
\hline 20 & 0.34 & 0.47 & $3.33 \mathrm{e}-3$ & $4.714 \mathrm{e}-3$ & $1.079 \mathrm{e}-3$ & $9.83 \mathrm{e}-4$ & $1.595 \mathrm{e}-4$ & $3.05 \mathrm{e}-4$ \\
\hline
\end{tabular}


While the estimated deformation is,

$$
\hat{\phi}(x, y, z)=\left(\begin{array}{lll}
4.9562 & 0.1211 & 0.2492 \\
0.3604 & 5.0734 & 0.2189 \\
0.3729 & 0.5817 & 4.8887
\end{array}\right)\left(\begin{array}{l}
x \\
y \\
z
\end{array}\right)+\left(\begin{array}{c}
4.9897 \\
10.0396 \\
15.0480
\end{array}\right)
$$

The accuracy of the estimated deformation field is assessed by computing the standard deviation of the estimated deformation field for $S$. For each point, the displacement field on $S$ is defined as $T_{\phi}(u, v)=\phi(S(u, v))-S(u, v)$. The displacement field $T_{\phi}$ and the estimated displacement field $T_{\hat{\phi}}$ are color-encoded and illustrated in 2. We first normalize each channel, $(x, y, z)$, of $T_{\phi}$ and $T_{\hat{\phi}}$ to be between 0 and 1 . Then $(x, y, z)$ are represented by $(R, G, B)$ colors. Higher values of error in any of the 3 channels/directions will have a higher brightness in the corresponding color.

As evident, the estimated deformation is very accurate. The distortion of the conformal structure caused by $\phi$ in the above experiment is very large, for example, it changes an angle from $90^{\circ}$ to $63^{\circ}$ in the above experiment. Even with such big distortions of the conformal structure, our method is able to quite accurately recover this deformation. This illustrates the robustness of our algorithm.

\subsection{Real Deformations Between Anatomical Surfaces}

We also tested our algorithm on subcortical surfaces, specifically, the human hippocampus surface extracted from MR brain scans. We applied our algorithm to several distinct hippocampal surfaces to estimate the deformation between pairs of them. This deformation can be used in assessing the shape differences between them and assess the asymmetry between the left and right hippocampi for a subject with epilepsy or schizophrenia or other pathologies.

Figure 3 illustrates the conformal representation of a hippocampal surface. The matching results are illustrated in figure 4 We match the first hippocampal surface to other three. In order to illustrate the correspondence, we color encode the $\mathrm{z}$ value of the first surface and map the color to other surfaces by the matching deformation. Each surface has about 5000 faces, and it takes about 90 seconds to compute their conformal representation and 200 seconds (on a pentium 4) for estimating the $3 \mathrm{D}$ deformation.

\section{Conclusions}

In this paper, we introduced a novel method for 3D surface matching using conformal representations, which is based on Riemann surface theories. All orientable surfaces can be mapped conformally to canonical spaces and represented by conformal factor and mean curvature. The representation intrinsically and continuously depends on the geometry of the surface. Then, the 3D surface matching problem is converted to matching of $2 \mathrm{D}$ conformal representations.

In comparison to other matching methods, our new method is intrinsic and efficient. We presented several synthetic and real data examples depicting the 


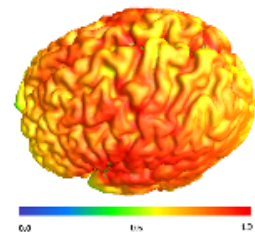

(a) conformal factor on (b)conformal factor (c) the brain surface.

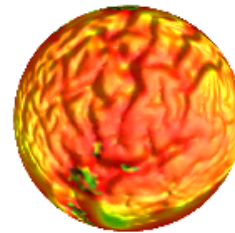

on the sphere.

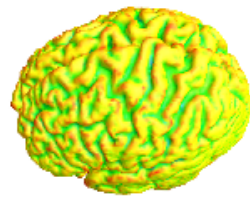

(c) Mean curvature on (d) Mean curvature the brain surface.

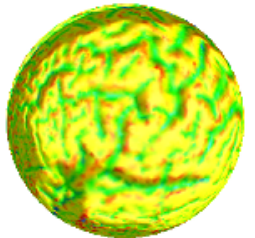

on the sphere.

Fig. 1. Color encoding of the conformal factor on (a) the cortex, (b) on the canonical domain - the sphere; Color encoding of the Mean curvature on (c) the cortex and (d) the cortex conformally mapped to the sphere.

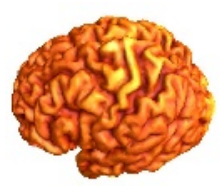

(a) Original brain surface
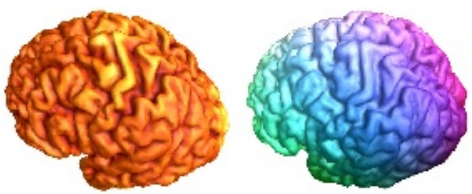

(b) Deformed brain (c) Displacement field(d) Displacement field surface

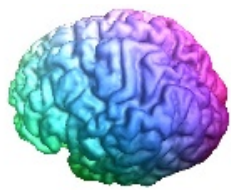

causod by $\hat{\phi}, T_{\hat{s}}$.

Fig. 2. Estimated deformation using the conformal representation. (a) Original cortical surface, (b) Synthetically deformed cortex. (c) and (d) depict color encoded deformation fields $\phi((x, y, z)$ corresponding to $(R, G, B))$ and $\hat{\phi}$ applied to (a) respectively.

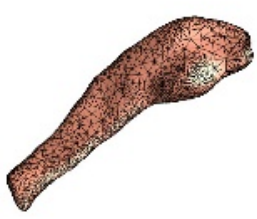

(a) Hippocampus surface
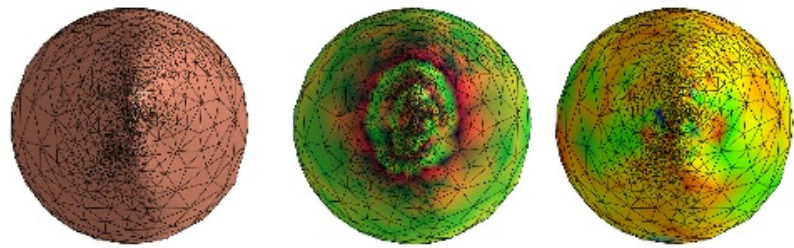

(b) Conformal Mapping(c) Conformal Factor (d) Mean curvature

Fig. 3. Conformal factor and Mean curvature maps of the hippocampal surface. (a) Hippocampal surface, (b) Conformal mapping of (a) to a sphere, (c) \& (d) color coded, conformal factor map and Mean curvature map respectively.

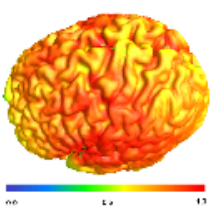

(a) conformal factor on the brain surlice.

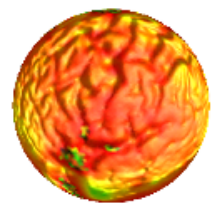

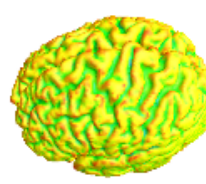

(c) Mean curvature o the brain surliace.

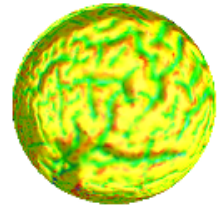

(d) Mean curvatiure on the sphere.

Fig. 4. Different hippocampal surfaces extracted from MRI images. The matching results are color encoded (see text for details). The regions of the same color exhibit the correspondence obtained from the matching algorithm. 
accuracy of the estimated deformation between the source and target shapes. The achieved accuracy was quite high and comparable to those achieved by other methods reported in literature but at a much smaller computational expense. Our future work will focus on generalizing the conformal representations to surfaces with arbitrary genus.

Acknowledgements. This research was in part supported by the NIH grant RO1 NS42075 to BCV. Authors thank Professor C. M. Leonard of the UF-MBI for the hippocampal data.

\section{References}

1. H. D. Tagare. Shape-based nonrigid correspondence with application to heart motion analysis. In IEEE Trans. Medical Imaging, volume 18, pages 570-578, 1999.

2. B. B. Kimia C. M. Cyr, T. Sabestian. 2d to 3d registration based on shape matching. In IEEE MMPIA, 2000.

3. M. Frenkel and R. Basri. Curve matching using the fast marching method. In EMMCVPR, pages 35-51, 2003.

4. B. C. Vemuri, A. Mitiche, and J. K. Aggarwal. curvature-based representation of objects from range data. In Image and Vision Computing, volume 4, pages 107-114, may 1986.

5. Paul J. Besl and Ramesh C. Jain. Three-dimensional object recognition. In ACM Computing Surveys, volume 17, pages 75-145, 1985.

6. A. Pentland and S. Scalroff. Closed-form solutions for physically based shape modeling and recognition. In IEEE Trans. on PAMI, volume 13, pages 715-729, 1991.

7. Dongmei Zhang and Martial Hebert. Harmonic shape images: A representation for 3d free-form surfaces based on energy minimization. In EMMCVPR, pages 30-43, 1999.

8. Michael Kazhdan and Thomas Funkhouser. Harmonic 3d shape matching.

9. V. Camion and L. Younes. Geodesic interpolating splines. In Proceedings of EMMCVPR 2001, pages 513-527, 2001.

10. A. Trouve. Diffeomorphisms groups and pattern matching in image analysis. In International Journal of Computer Vision, volume 28, pages 213-221, 1998.

11. P. Thompson and A. Toga. A surface-based technique for warping 3-dimensional images of the brain. In IEEE Trans. Medical Images, volume 15, pages 402-417, 1996.

12. P. M. Thompson, M. S. Mega, C. Vidal, J. L. Rapoport, and A. W. Toga. Detecting disease-specific patterns of brain structure using cortical pattern matching and a population-based probabilistic brain atlas. In 17th International Conference on Information Processing in Medical Imaging (IPMI2001), volume 18, pages 488501, 2001.

13. Csernansky, J., Joshi, S., Wang, L., Haller, J., Gado, M., Miller, J., Grenander, U., Miller, and M. Hippocampal morphometry in schizophrenia via high dimensional brain mapping. In Proceedings National Academy of Sciences, pages 11406-11411, 1998. 
14. G. E. Christensen, S. C. Joshi, and M. Miller. Volumetric transformation of brain anatomy. In IEEE TMI, volume 16, pages 864-877, 1997.

15. Carole Twining and Stephen Marsland. Constructing diffeomorphic representations of non-rigid registrations of medical images. In 18th International Conference on Information Processing in Medical Imaging (IPMI2003), volume 20, pages 413-425, 2003.

16. D. L. Collins, C. J. Holmes, T. M. Peters, and A. C. Evans. Automatic 3d model based neuroanatomical segmentation. In Human Brain Mapping, volume 3, pages 190-208, 1995.

17. S. Pentland, S. Angenent, A. Tannenbaum, R. Kikinis, G. Sapiro, and M. Halle. Conformal surface parameterization for texture mapping. In IEEE Transaction on Visualization and Computer Graphics, volume 6, pages 181-189, 2000.

18. X. Gu, Y. Wang, T.F. Chan, P.M. Thompson, and S.T. Yau. Genus zero surface conformal mapping and its application to brain surface mapping. In IPMI2003, pages $172-184$. 